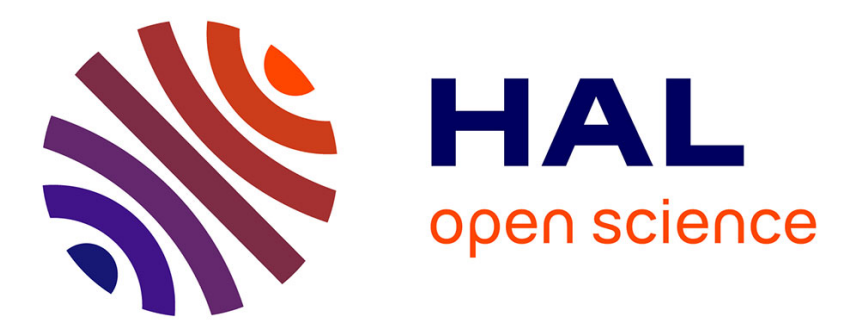

\title{
Prognostics of Health Status of Multi-component Systems With Degradation Interactions.
}

Wael Hafsa, Brigitte Morello, Christophe Varnier, Kamal Medjaher, Noureddine Zerhouni

\section{- To cite this version:}

Wael Hafsa, Brigitte Morello, Christophe Varnier, Kamal Medjaher, Noureddine Zerhouni. Prognostics of Health Status of Multi-component Systems With Degradation Interactions.. International Conference on Industrial Engineering and Systems Management, Oct 2015, Seville, Spain. hal-02868215

\section{HAL Id: hal-02868215 https://hal.science/hal-02868215}

Submitted on 15 Jun 2020

HAL is a multi-disciplinary open access archive for the deposit and dissemination of scientific research documents, whether they are published or not. The documents may come from teaching and research institutions in France or abroad, or from public or private research centers.
L'archive ouverte pluridisciplinaire HAL, est destinée au dépôt et à la diffusion de documents scientifiques de niveau recherche, publiés ou non, émanant des établissements d'enseignement et de recherche français ou étrangers, des laboratoires publics ou privés. 


\title{
Prognostics of Health Status of Multi-component Systems With Degradation Interactions
}

\author{
(presented at the $6^{\text {th }}$ IESM Conference, October 2015, Seville, Spain) (C) $\mathrm{I}^{4} \mathrm{e}^{2} 2015$
}

\author{
Wael Hafsa, Brigitte Chebel-Morello, Christophe VARNIER, Kamal MEDJAHER, Noureddine ZERHOUNI \\ FEMTO-ST institute, UMR CNRS 6174 - UFC / ENSMM / UTBM \\ Automatic Control and Micro-Mechatronic Systems Departement, \\ 24, rue Alain Savary, 25000 Besançon, France \\ Email addresses: \{wael.hafsa, brigitte.morello, christophe.varnier, kamal.medjaher, noureddine.zerhouni\}@femto-st.fr
}

\begin{abstract}
Many models and methodologies in order to predict the remaining useful life (RUL) of a critical component are investigated nowadays. However, estimating remaining useful life of multi-component systems is still an under explored area, especially when there are interdependencies among the components of these systems. Practically, prognostics can be quite complicated when there is absence of prior knowledge about these interactions. To optimize the availability and reliability of the system, it is required to embed a dependency model that implements component strength and the component's health status. In this paper, a novel approach is proposed in order to emphasize the importance of interactions between complex system's components in the RUL's calculating. The effectiveness of the approach is judged by applying it to numerical studies in order to estimate system's remaining useful life.
\end{abstract}

\section{INTRODUCTION}

Nowadays, the development of industrial systems is increasingly complex due to new technologies, and industrial manufacturing systems are becoming more complicated. Complex system may be defined as a coherent system composed of many interacting entities. Controlling, managing and keeping such a system in a good health status isn't an easy task. This topic has received more attention from academic researchers and manufactures in the past decade. In fact, many research works have been developed on reliability of complex systems and their maintenance [11], [10], [1]-[2]. Prognostics is considered as a key process in maintenance strategies in order to predict the RUL (remaining useful life) and the performance of multi-component and networked systems, like aircraft, cars and mechanical machinery. Prognostics and Health Management (PHM) is an emerging discipline which links studies of failure mechanisms and life cycle management [3]. According to ISO:13381-1, prognostics is defined as the "estimation of time to failure and risk for one or more existing and future failure modes" [4]. The use of prognostics methods in maintenance in order to predict the RUL is receiving more attention over the past years. Many experts expect that effective prognostics will result (i) in reduced numbers and severity of failures, especially failures in the field, (ii) optimizing operational performance, (iii) extending the time between needed maintenance activities and reducing life-cycle costs. However, the use of these techniques in maintenance decision making and optimization in multi-component systems is still an under explored area due to the complexity of these systems which introduces additional interdependencies between components. In fact, complex systems are handled differently from single-component systems because we need a full understanding of interactions between components, and neglecting them leads to inefficient prognostics. Ancient approaches are reduced to consider the minimum of critical components RULs and to neglect interdependencies between components in order to avoid any possible system's shutdown. Nevertheless, many researches have reported that the assumption of independence is impractical. In fact, Sun et al. [7] notice that this assumption leads frequently to errors in estimating the system's RUL in real applications. Therefore, the decision making based on the resulting remaining useful life should be established in a predictive maintenance policy which highlights dependency between system components. Interdependencies between complex system components have been extensively studied in operations research literature for the past few decades. Murthy and Nguyen [5] indicate that the analysis of maintenance policies with failure interactions is an open problem. More recently, Nicolai and Dekker [6] have given a thorough overview of optimal maintenance policies for complex systems with dependency between components (i.e. stochastic, structural or economic dependence). However, no models that use prognostics or a prediction of Remaining Useful Life are mentioned. This paper focuses on stochastic dependence, where the degradation or the failure of one component influences the life time distribution of other components in the system, i.e. if a component degrades over time (due to wear, environmental variables or use conditions), it has an impact on the performance of other components by means of accelerating their degradation. In that context, Bian and Gebraeel focus on how degradation-based sensor signals associated with the components evolve over time, using stochastic models characterizing the natural degradation rates and the effects of interactions on the degradation rate [8]-[9]. $\mathrm{Wu}$ et al. [10] present a method to consider stochastic loading and strength degradation for computing the time-dependent reliability of a structural component. Besides, Van Horenbeek and Pintelon [11] present a dynamic predictive maintenance policy for complex systems that minimizes the long-term mean maintenance cost per unit time. This method is based on prognostics information while considering different component dependencies (i.e. economic, structural and stochastic). Moreover, Ribot [12]-[13] aims at improving classical approach to estimate the RUL of a complex system. Mainly, Ribot's approach has an advantage of better system modeling, when there is absence of components dependency. Our work differs from the papers mentioned above in two folds, as shown in Fig. 1: i) Presenting a stochastic dependency model for degradation rate 
interactions, ii) Proposing a prognostics method to calculate the system's RUL considering the degradation rate interactions between system's components. The remainder of the paper is

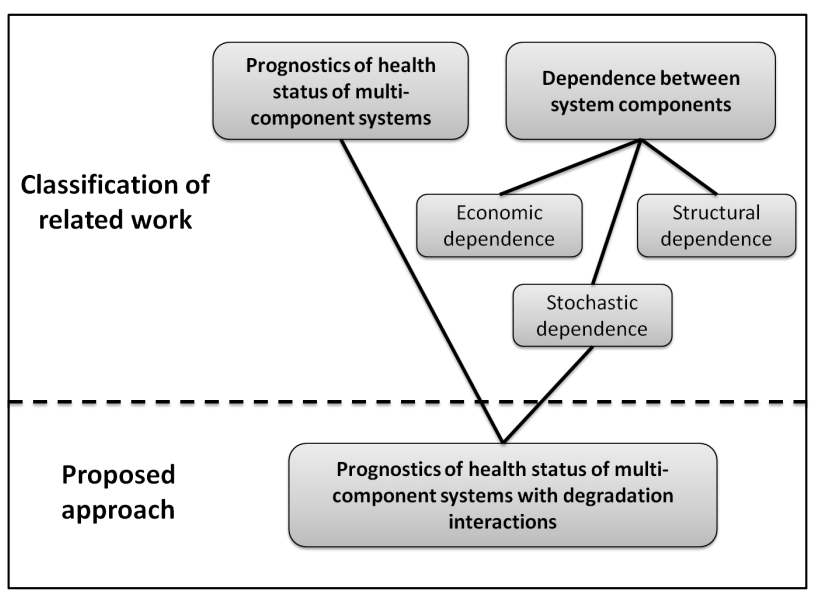

Fig. 1: Position of the proposed approach

organized as follows: In section 2, we describe the prognostics method. Sequentially, we present a stochastic dependency model in details with necessary assumptions. Under these assumptions, we prove in section 4 our approach bu presenting a numerical studies. Conclusions are presented in section 5.

\section{PROGNOSTICS METHOD}

\section{A. Introduction}

Prognostics basically consists in estimating at time $t_{i}$ the time $t_{i+1}$ of the system failure. A prognostics function is defined to assess the probability associated with each possible future failure for the system. This probability is obtained from a set of aging models available in the current operational mode of the system. We assume that a set of aging models is available for each component in the system. These models represent the knowledge available about component's wear. They may indicate an average value of the next component's failure as the mean time to failure (MTTF) [12]-[13]. We consider as an example, a system of two interconnected components $C_{l}$ and $C_{2}$ with a set of failure probability density functions denoted by $f_{C 1}(t)$ and $f_{C 2}(t)$ respectively, as in Fig. 2, where the Weibull model represents the probability density function.

\section{B. System's remaining useful life}

We consider a system of $n$ interconnected components $C_{1}, C_{2}, \ldots, C_{n}$ with a set of aging models denoted by $S_{1}(t)$, $S_{2}(t), \ldots, S_{n}(t)$ respectively. $S_{i}(t)$ is defined without taking into account interactions between components, in other word, it represents the component's own model. Failure probability is determined for each component from its aging model selected in the current operating mode. Let $f_{i}$ denote the probability density function (pdf) representing the failure probability of a component $i$ in its current operating mode, $P_{\max }$ be the maximal failure probability threshold acceptable for the component $C_{i}$. The remaining useful life of $C_{i}$ consists in determining the time $t_{c}$ for which the failure probability has reached the threshold $P_{\max }$, as in (1).

$$
R U L\left(C_{i}\right)=t_{c} \text { such that } \int_{0}^{t_{c}} f_{c}(t) d t=P_{\max }
$$

The date of the next system failure is then calculated by :

$$
t_{i+1}=t_{i}+\min \left(\operatorname{RUL}\left(C_{i}\right)\right) \text { where } i \in[1, n]
$$

\section{Weibull model}

The difficulty is to establish a common prognostics representation for each type of component. This representation should be as flexible as possible to represent the failure probability density functions of each component in the system.

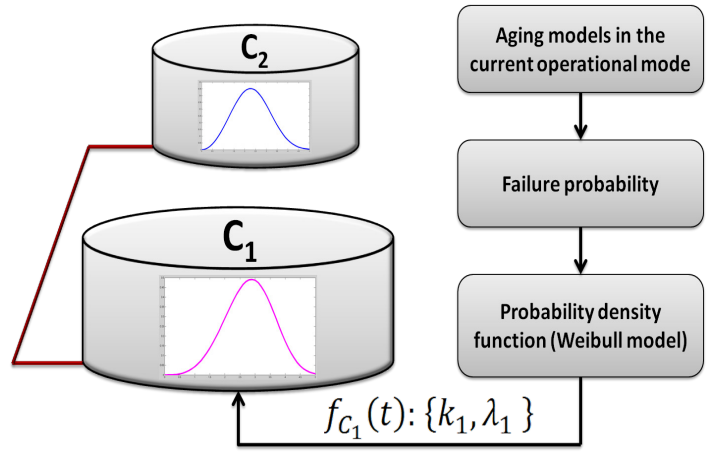

Fig. 2: Component's representation with the Weibull model

The probabilistic Weibull model is often used in the field of survival and failure analysis, reliability and systems life data analysis [14]-[12]. The Weibull probability density function is:

$$
W(t, \kappa, \lambda, \theta)=\frac{\kappa}{\lambda}\left(\frac{t-\theta}{\lambda}\right)^{\kappa-1} e^{-\left(\frac{t-\theta}{\lambda}\right)^{\kappa}}
$$

where $t \geq 0, \kappa \geq 0, \lambda \geq 0$, and $\theta \geq 0$. $\kappa$ represents the shape parameter of the distribution, $\lambda$ defines the scale parameter and $\theta$ determines its location on the time axis. These parameters define the probability distribution and model component aging signs. We assume that prognostics were done in the same time for all system's components, this translates into $\theta=0$. For a given component $C_{i}$ and a given probability threshold $P_{\max }$, the remaining useful life is:

$$
R U L\left(C_{i}\right)=t_{c} \text { such that } \int_{0}^{t_{c}} \frac{\kappa}{\lambda}\left(\frac{t}{\lambda}\right)^{\kappa-1} e^{-\left(\frac{t}{\lambda}\right)^{\kappa}} d t=P_{\max }
$$

\section{STOCHASTIC DEPENDENCY MODEL FOR DEGRADATION RATE INTERACTIONS}

In this section, we present a stochastic methodology by modeling the degradation interactions effects of interdependent components $C_{1}, C_{2}, \ldots, C_{n}$ knowing its failure cumulative distribution functions $F_{1}\left(t, \kappa_{1}, \lambda_{1}\right), F_{2}\left(\left(t, \kappa_{2}, \lambda_{2}\right)\right), \ldots, F_{n}\left(\left(t, \kappa_{n}, \lambda_{n}\right)\right)$ (6). 


\section{A. Cumulative distribution function $C D F$}

To facilitate the analysis of degradation interactions between system components, each component will be represented by its cumulative distribution, as in Fig. 3. Moreover, CDF will allows us to fully note the degradation of the component's health state. In probability theory and statistics, the cumulative distribution function of a continuous random variable $X$ can be expressed as the integral of its probability density function $f_{X}$ as follows:

$$
F_{X}(t)=\int_{-\infty}^{x} f_{X}(t) d t
$$

The cumulative distribution function for the Weibull distribution is:

$$
F(x ; \kappa ; \lambda)=1-e^{-\left(\frac{x}{\lambda}\right)^{\kappa}}
$$

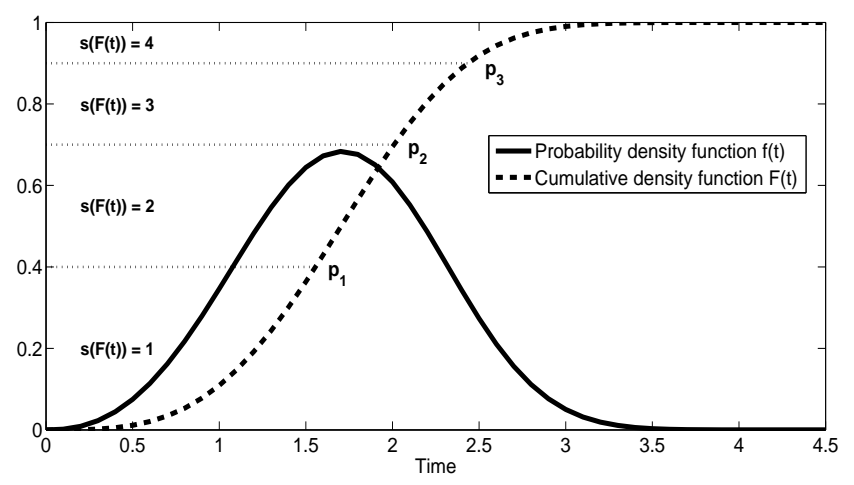

Fig. 3: CDF of the Weibull distribution with different degradation states

\section{B. Component's degradation states}

Interaction considers that the failure of one component induces failure of all other interdependent components. We refer to the former as the "influencing component" and the latter as the "affected component". The transition of an influencing component, say $C_{i}$ to a more degraded state increases the degradation rate of the affected component, say $C_{a}$ by an amount $\rho_{i \rightarrow a}$ which depend on the degradation states of the influencing component. We can take as an example "the gear train" which is a mechanical system formed by mounting gears on a frame so that the teeth of the gears engage. The degradation of a gear teeth may cause the degradation of the next one, which may increase the looseness of the smooth transmission of rotation from one gear to the next. In this example, the system is the rotation transmission system. Thus, based on the value of failure probability, a component $C_{i}$ can be classified into different degradation states, e.g., "nominal", "degraded", "critical", "failed" (see Fig. 3). Let $s_{i}(t)$ denotes the degradation state of component $C_{i}$, whereby $s_{i}$ is piecewise constant function, as in (7):

$$
s\left(F_{i}(t)\right)=\left\{\begin{array}{cc}
1 & F_{i}(t)<p_{i, 1} \\
2 & p_{i, 1} \leq F_{i}(t)<p_{i, 2} \\
3 & p_{i, 2} \leq F_{i}(t)<p_{i, 3} \\
4 & F_{i}(t) \geq p_{i, 3}
\end{array}\right.
$$

where $p_{i, 1}, p_{i, 2}, p_{i, 3}$ and $p_{i, 4}$ represent the probability thresholds for different degradation states. $s(F(t))$ represents the degradation state of the influencing component.

\section{Component's strength}

Most components degrade as they age or deteriorate as a result of some factors that termed as covariates, i.e., degree of use, amount of load or extent of stress they experienced. To illustrate this idea, we can take the example of health concerns of old people. As people age, poor resistance and strength contribute to falls, health problems and, in some cases, fatal complications. Let $R_{i}(t)$ represents the strength of component $C_{i} . R_{i}(t)$ is a random variable that decreases over time and it can be described by gamma process which reduces the reliability and accelerate the risk of failure and shortens its life service [10]. Wu et al. [10] define the component strength which drops below the load as : $R_{i}(t)=R_{0}-x(t)$, where $R_{0}$ represents the initial strength and $x(t)$ is the component strength's deterioration over time, as in Fig (4).

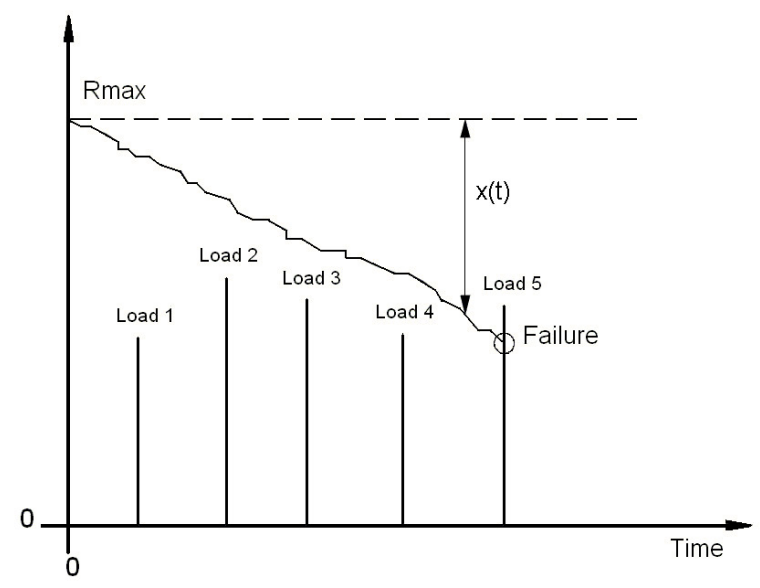

Fig. 4: Deterioration of component strength

\section{Degradation rate of interactions}

We denote the degradation rate of component $C_{i}$ at time t by $r_{i}(t)$. We assume that $r_{i}(t)$ is composed of two parts: (i) Component's strength, (ii) component's degradation state. Thus, our proposed degradation rate of component $C_{i}$ can be written in the following form: $r_{i}(t)=r_{i}\left[t ; R_{i}(t), s(F(t))\right]$. To facilitate model development, we assume that the degradation rates of system components can be expressed as:

$$
r(t)=\frac{\rho_{i \rightarrow a}}{R(t)}
$$

Degradation occurs when the impact of influencing component degradation is higher than affected component's strength. Generally, the impact of influencing component's degradation tends to increase, while the component strength tends to decrease due to deterioration induced by higher than expected loads or fatigue damage. 


\section{E. Weibull model with degradation interactions}

If the quantity $t$ is a "time-to-failure", the Weibull distribution described in (3) gives a distribution for which the failure rate is proportional to a power of time. The shape parameter $\kappa$, is that power plus one [15], and so this parameter can be interpreted directly as follows :

- A value of $\kappa<1$ indicates that the failure rate decreases over time.

- A value of $\kappa=1$ indicates that the failure rate is constant over time.

- A value of $\kappa>1$ indicates that the failure rate increases with time. This happens if there is an "aging process i.e., the degradation states of influencing components", or parts that are "more likely to fail i.e., component strength" as time goes on.

Moreover, the coefficient of variation depend only on the shape parameter. Thus, The degradation evolution of the cumulative distribution function $F_{i}(t)$ can be modeled as in (9):

$$
F\left(t ; \kappa^{\prime} ; \lambda\right)=1-e^{-\left(\frac{t}{\lambda}\right)^{\kappa \cdot r(t)}}
$$

where $\kappa^{\prime}=\kappa \cdot r(t)$. Equation (9) describes our stochastic dependency model which characterizes the effects of degradation interaction arising from other components. For the aim of this model, we consider the following assumptions:

1. $\kappa$ represents the original degradation rate of the component $C_{a}$ without taking into account the effect of dependency between system components, i.e., the strength of the "affected component" is maximal $R_{a}(t)=R_{a \operatorname{Max}}$ and the degradation state of "the influencing component" is nominal $s\left(F_{i}(t)\right)=1$.

2. The degradation rate of a component $C_{i}$ is a linear function of its component strength and the degradation states of other influencing components, precisely $r(t)=\frac{\rho_{i, a}}{R(t)}$.

3. Degradation is triggered when the degradation of influencing component exceeds affected component's strength that is liable to deterioration, i.e., $r(t)>0$.

4. The whole system is inspected simultaneously at a fixed time, and all inspection actions are perfect and instantaneous.

Figure 5 provides an example of the impact of component degradation interactions from a system that exhibit stochastic dependency.

\section{APPLICATION}

\section{A. System description}

Our application is interested in ski lift. We illustrate our approach by studying the Lorry system which is a must for the good functioning of a ski lift, see Figure (6). Lorry is a tension system that enables to maintain cable tension almost constant on the driving pulley. In this case, an engine unit including the driving pulley is installed on a carriage which can move in the desired direction, forward or backward. In fact, the cable's length for a ski lift is made to change over

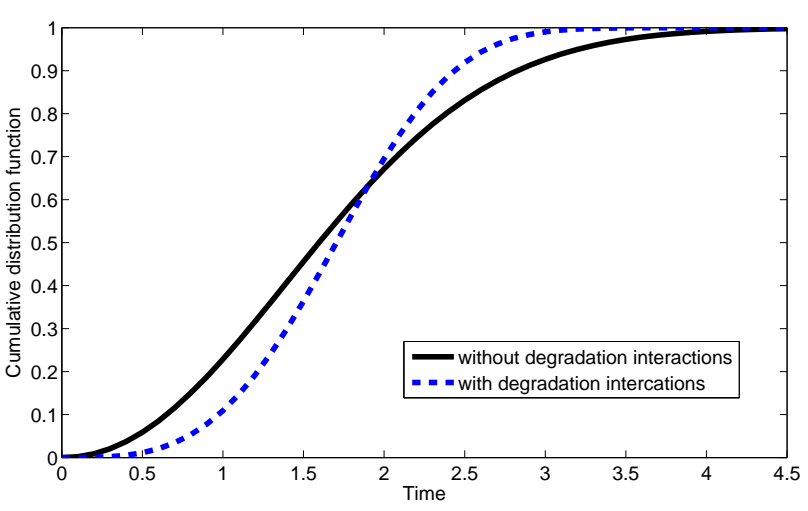

Fig. 5: Example of cumulative distribution function with degradation interactions

time, load and temperature. The cable's tension is mostly adjusted by one or two hydraulic jacks, which are powered by a hydraulic pump. We assume that engine of hydraulic pump and jacks present the most critical components of the system where engine is the influencing component and jacks represent the affected component. Thus, we consider a system

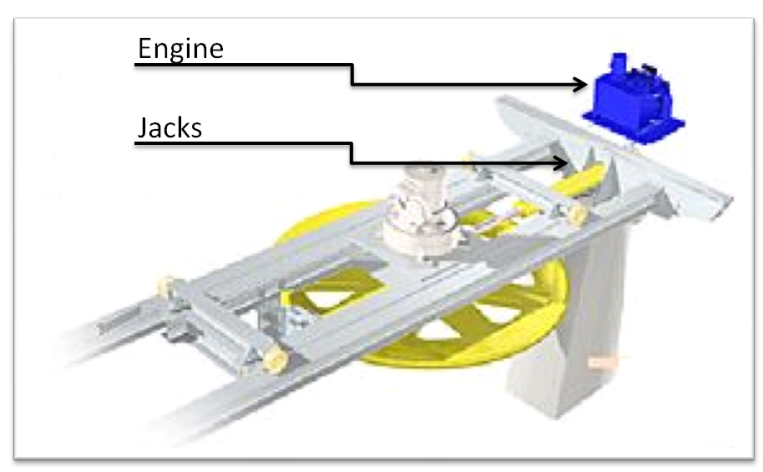

Fig. 6: Lorry system

of 2 interconnected components $C_{\text {engine }}$ and $C_{\text {Jacks }}$ with a set of failure probability density functions denoted by $f_{\text {engine }}(t)$ and $f_{\text {Jacks }}(t) . \lambda$ and $\mathrm{k}$ represent respectively the scale and shape parameters, as in (10), and $P_{\text {Max }}$ defines the probability threshold.

$$
f_{\text {engine }}:\left\{\begin{array}{c}
k_{e}=2.6 \\
\lambda_{e}=2 \\
P_{M a x, e}=0.9
\end{array} \quad f_{\text {Jacks }}:\left\{\begin{array}{c}
k_{J}=2.1 \\
\lambda_{J}=1.9 \\
P_{M a x, J}=0.9
\end{array}\right.\right.
$$

Th system components are assumed to be interdependent and their degradation interactions are manifested in their cumulative distribution functions. For the purpose of this study, we simulate cumulative distribution functions for all two components using equation (6). To simulate degradation process for different scenarios, we consider two experimental settings: (i) In the first experiment, our model is equivalent to models that do not consider any interactions between components and assume components independence. (i) Next, for the second experiment, we study degradation interactions between system's components. 
To facilitate our simulation, we start with a baseline setup. The initial strength of Jacks is $R_{J \max }=750 M P a$, and it decreases over time as shown in Fig. 7. Next, we note that

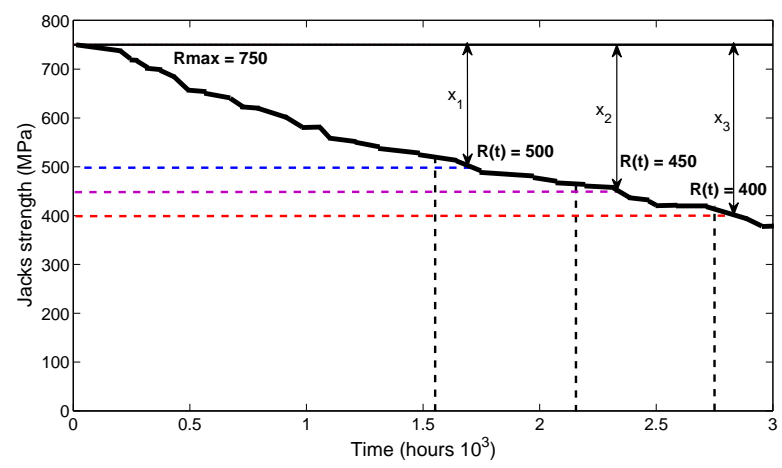

Fig. 7: Deterioration of Jacks strength

$\rho_{\text {engine } \rightarrow \text { Jacks }}$ represents the engine's degradation amplitude, i.e., the incremental change in the jacks degradation when engine switches to a more severe state. Engine degradation states are described in Fig. 8 where we notice four degradation states, nominal, degraded, critical and failed according to (11). For these degradation states, the values of probability thresholds $p_{\text {engine }, 1}, p_{\text {engine }, 2}$ and $p_{\text {engine }, 3}$ are respectively $0.4,0.7$ and 0.9 .

$$
s\left(F_{\text {engine }}(t)\right)=\left\{\begin{array}{cc}
1 & F_{\text {engine }}(t)<0.4 \\
2 & 0.4 \leq F_{\text {engine }}(t)<0.7 \\
3 & 0.7 \leq F_{\text {engine }}(t)<0.9 \\
4 & F_{\text {engine }}(t) \geq 0.9
\end{array}\right.
$$

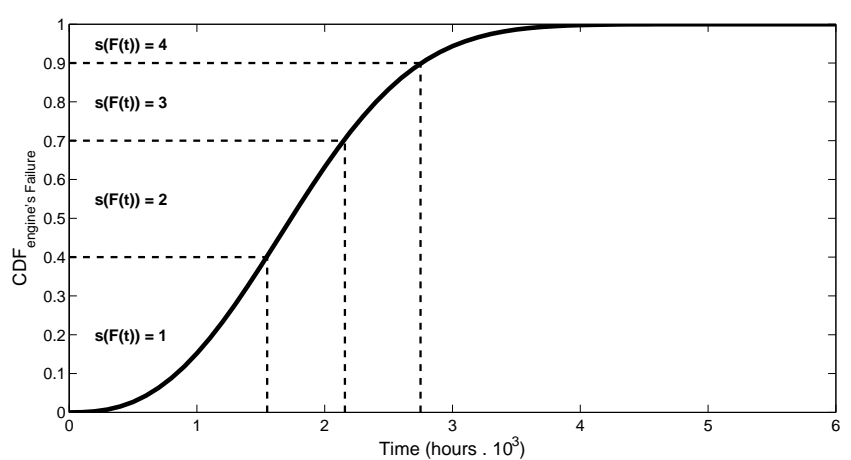

Fig. 8: engine's degradation states

To investigate the impact of different degradation magnitudes, we consider three levels of degradation interactions $r_{J}$ $=(1.6 ; 2 ; 3)$, see table I. In other words, this allows us to study the impact of different amplitudes of degradation interactions.

\section{B. Results}

With the increase of degradation rate $r_{J}(t)$, the value of the $R U L_{\text {system }}$ gradually decreases which can be shown in table II. In the first scenario where there are no interactions between system components, the system remaining useful life depends on the engine's RUL, i.e., engine represents the most
TABLE I: Degradation interactions rates

\begin{tabular}{|c||c||c|}
\hline$\rho_{\text {engine } \rightarrow \text { Jacks }}(\mathrm{MPa})$ & $R_{\text {Jacks }}(1000)(\mathrm{MPa})$ & $r_{J}$ \\
\hline 750 & 750 & 1 (No interaction) \\
\hline 800 & 500 & 1.6 \\
\hline 900 & 450 & 2 \\
\hline 1200 & 400 & 3 \\
\hline
\end{tabular}

TABLE II: Simulation results

\begin{tabular}{|c||c||c||c|}
\hline$r_{J}$ & $R U L_{\text {Jacks }}$ & $R U L_{\text {Engine }}$ & $R U L_{\text {System }}$ \\
\hline 1 & 1826.4 & 1750 & 1750 \\
\hline 1.6 & 1435.3 & 1750 & 1435.3 \\
\hline 2 & 1317.4 & 1750 & 1317.4 \\
\hline 3 & 1168.9 & 1750 & 1168.9 \\
\hline
\end{tabular}

critical component of the system. Taking into account the dependencies between the components changes the behavior of the system's RUL, and we clearly see that it becomes dependent of the value of the jacks RUL. Figures (9) and (10) illustrate the change in the jacks failure probabilities.

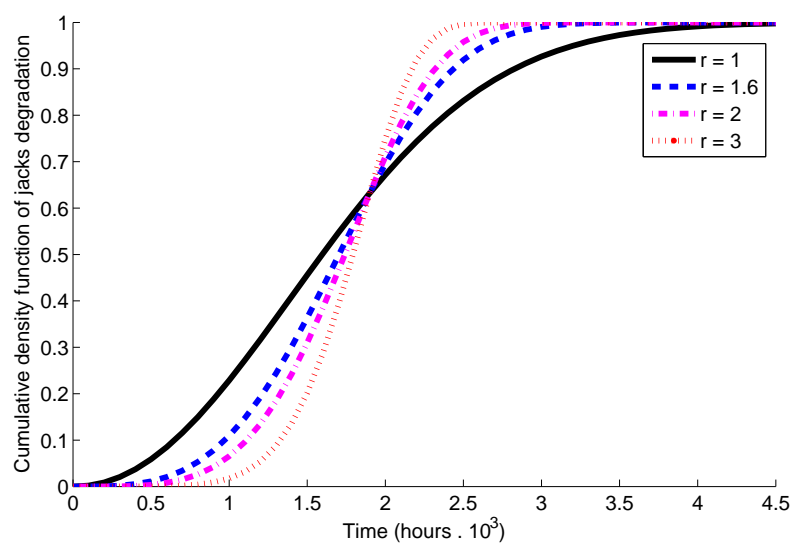

Fig. 9: CDF of jacks degradation interactions

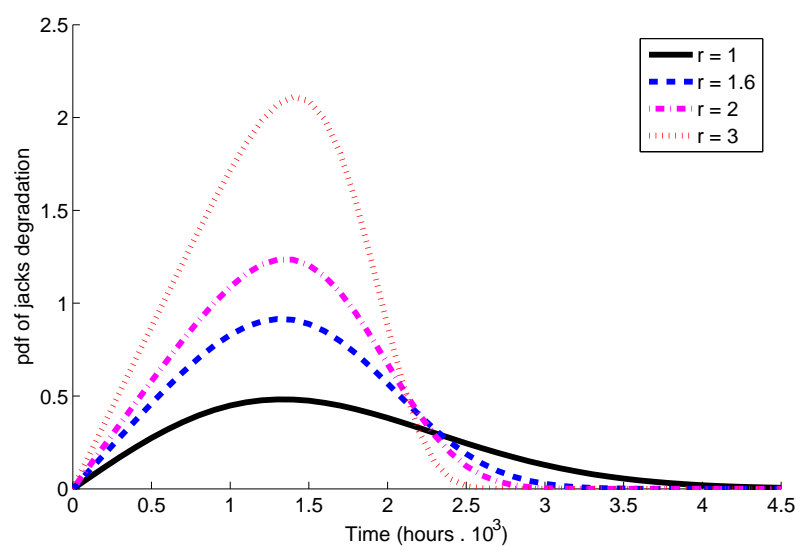

Fig. 10: Weibull model for jacks degradation interactions

Indeed, the high availability of systems such as aerospace, defense, petrochemical and automotive, is an important tar- 
get of the new developments in systems design technology knowing that the failure is generally very expensive. To make maintenance strategies more effective and to take into account the system's changing status, the maintenance action has to be done at the right time according to the system remaining useful life given by the prognostics process. This allows us to increase the time of availability, performance and reliability of the system. The stochastic dependency is included in our approach to make it more accurate and realistic. In fact, a Weibull model is developed to highlight the link between prognostic method and dependencies between systems components. The decision making determined from the resulting RUL should be established, taking into account dependency between system components. These interactions require to be integrated in degradation models by considering these data as input data.

\section{CONClusion}

In this paper, we study prognostics approach for multicomponent system which have failure interactions among them. The approach presented allows us in the first place to calculate the remaining useful life of system's components. Secondly, it permits to characterize the interactions among the degradation process of interdependent components where both the deteriorating component's strength and the health status of component are modeled as stochastic process. Hence, Our approach predicts the system's remaining useful life and demonstrates how the availability of a complex system is affected by its constituent components. Through numerical example, we demonstrate that failure interactions impacts prognostics results and these impacts will be intensified as the degradation interactions rate increases.

\section{ACKNOWLEDGMENT}

The authors would like to acknowledge the European Regional Development Fund who funded this work as a part of a project named MainPreSI realized within the framework of the European Territorial Cooperation program INTERREG IV A France - Switzerland.

\section{REFERENCES}

[1] Z. Zhang, S. Wu, B. Li, and S. Lee, (n, N) type maintenance policy for multi-component systems with failure interactions, International Journal of Systems Science, 46(6), 1051-1064, 2015.

[2] Z. Zhang, S. Wu, S. Lee, and J. Ni, Modified iterative aggregation procedure for maintenance optimisation of multi-component systems with failure interaction, International Journal of Systems Science 45(12), 2480-2489, 2014.

[3] S. Uckun, K. Goebel and P.J. Lucas, Standardizing research methods for prognostics. In Prognostics and Health Management, 2008. PHM 2008, International Conference on (pp. 1-10), IEEE, October 2008

[4] ISO13381-1, Condition monitoring and diagnostics of machines - prognostics - Part1: General guidelines, International Standard, ISO, 2004.

[5] D.N.P. Murthy and D.G. Nguyen, Study of a Multi-component System with Failure Interaction, European Journal of Operational Research, 21(3), 330-338, 1985.

[6] R.P. Nicolai and R. Dekker, Optimal maintenance of multi-component systems: a review (pp. 263-286). Springer London, 2008.

[7] Y. Sun, L. Ma, J. Mathew, W. Wang and S. Zhang, Mechanical systems hazard estimation using condition monitoring, Mechanical Systems and Signal Processing, 20(5), 1189-1201, 2006.

[8] L. Bian and N. Gebraeel, Stochastic framework for partially degradation systems with continuous component degradationrateinteractions, Naval Research Logistics (NRL), 61(4), 286-303, 2014.
[9] L. Bian and N. Gebraeel, Stochastic modeling and real-time prognostics for multi-component systems with degradation rate interactions. IIE Transactions, 46(5), 470-482, 2014.

[10] Y. Wu, L. Xie, N. Wu, and J. Li. Time-dependent reliability model of components with strength degradation based-on gamma process. In Reliability, Maintainability and Safety (ICRMS), 9th International Conference on (pp. 363-368), IEEE, June 2011.

[11] A. Van Horenbeek and L. Pintelon, A dynamic predictive maintenance policy for complex multi component systems, Reliability Engineering and System Safety, 120, 39-50, 2013.

[12] P. Ribot, Vers l'intgration diagnostic/pronostic pour la maintenance des systmes complexes (Doctoral dissertation, Universit Paul SabatierToulouse III), 2009.

[13] P. Ribot, Y. Pencol and M. Combacau, Diagnosis and prognosis for the maintenance of complex systems, in SMC (pp. 4146-4151), October 2009.

[14] D. Humphrey, W. Schawlee, P. Sandborn and D. Lorenson, Utilization Life of Electronic Systems - Aging avionics usable life and wear-out issues, proceedings of world aviation congress, Phoenix, AZ, USA, November 2002.

[15] http://en.wikipedia.org/wiki/Weibull_distribution, April 2015. 